\title{
The Stellar Populations of M32: Resolving the nearest elliptical with HST ACS/HRC
}

\author{
Antonela Monachesi ${ }^{1}$, S.C.Trager ${ }^{1}$, Tod R. Lauer ${ }^{2}$, Wendy \\ Freedman $^{3}$, Alan Dressler ${ }^{3}$, Carl Grillmair ${ }^{4}$ \& Kenneth Mighell ${ }^{2}$ \\ ${ }^{1}$ Kapteyn Astronomical Institute, P.O. Box 800, 9700 AV, Groningen, the Netherlands \\ email: monachesi@astro.rug.nl \\ ${ }^{2} \mathrm{NOAO},{ }^{3} \mathrm{OCIW},{ }^{4}$ Caltech
}

\begin{abstract}
We present the deepest colour-magnitude diagram (CMD) of M32 to date, obtained from deep (F435W, F555W) photometry of HST ACS/HRC images. Due to the high resolution of our images, the small photometric errors, and the completeness level of our data we obtain the most detailed resolved photometric study of M32 to date. The CMD of M32 displays a wide colour distribution of red giant branch stars, mainly due to a metallicity spread, a strong red clump and bright asymptotic giant branch stars. The detection of a "blue plume" in M32 indicates the presence of a very young stellar population. There is not a noticeable presence of blue horizontal branch stars, suggesting that an old population with $[\mathrm{Fe} / \mathrm{H}]<-1.5$ does not significantly contribute to the light or mass of M32 in our observed fields.
\end{abstract}

Keywords. Local Group, galaxies: individual: M32, galaxies: elliptical and lenticular, cD, galaxies: stellar content, stars: evolution.

\section{Introduction}

The Local Group galaxy Messier 32 (M32) is a small satellite of M31 and the nearest elliptical galaxy with properties similar to giant ellipticals. Given its proximity, M32 can be studied by both its integrated spectrum and the photometry of its resolved stars possibly down to the main-sequence turn-off (MSTO). It is therefore a vital laboratory to test the applicability of stellar population models to more distant galaxies, which can only be studied by the spectra of their integrated light.

Despite the fact that M32 is the most extensively observed and studied elliptical, its star formation history (SFH) remains a puzzle. M32 appears to have had one or more relatively recent episodes of star formation (within the last 3 Gyr: e.g., O'Connell 1980; Rose 1985; Trager et al. 2000; Coelho et al. 2009). O'Connell (1980) concluded that models fail to reproduce M32 with a single old-age and solar-metallicity population. These conclusions however rest on painstaking and controversial spectral analysis of their integrated light. In contrast, the most direct information about a stellar population comes from applying stellar evolution theory to colour-magnitude diagrams (CMDs). Thus the only way to derive the SFH of M32 and test conclusions so far based solely on integrated colours is to obtain deep CMDs that reach the MSTO of M32. Previous photometric works have only obtained information from the brighter stars of M32, prevented from observing fainter stars due to the crowding of M32. Davidge \& Jensen (2007) found AGB stars suggestive of a $\sim 3$ Gyr old, intermediate-age population. Grillmair et al. (1996) demonstrated the composite nature of the CMD of M32, concluding that the broad color distribution of the giant stars implies an intrinsically wide metallicity distribution. To further investigate the stellar content of M32, and with the primary goal of deriving a complete SFH of this enigmatic galaxy, we were awarded 64 orbits of the Hubble Space 
Telescope (HST) to observe the MSTO of M32 with the High-Resolution Channel of the Advanced Camera for Surveys (ACS/HRC).

\section{Observation, data reduction and photometry}

We obtained deep $B$ - and $V$-band imaging of two fields near M32 using ACS/HRC during Cycle 14 (Program GO-10572, PI: Lauer). The ACS F435W $(B)$ and $F 555 W$ $(V)$ filters were selected to optimize detection of MSTO stars over the redder and more luminous stars of the giant branch. The M32 HRC field (hereafter F1) was centred on a location $\sim 110^{\prime \prime}$ south (the anti-M31 direction) of the M32 nucleus, which represents the best compromise between minimizing the extreme crowding in M32 and maximising the contrast of M32 against the M31 background population. Even at the location of F1, M31 contributes $\sim 1 / 3$ of the total light, thus it was critical to obtain a background field (hereafter F2) at the same isophotal level in M31 to allow for the strong M31 contamination to be subtracted from the analysis of the M32 stellar population. F2 was located $327^{\prime \prime}$ from the M32 nucleus. Fig. 1 (left panel) shows the location of both the F1 and F2 fields. Each field was observed for 16 orbits in each of the $F 435 W$ and $F 555 W$ filters for a total program of 64 orbits. The data set for each of the four filter/field combinations comprises 32 exposures that were combined in an iterative procedure designed to detect and repair cosmic-ray events, hot pixels, and other defects, with a Nyquist-sampled summed image as the final product (Lauer 1999). The combined images of F1 and F2 fields used for analysis thus have a pixel scale of $0.0125^{\prime \prime}$, which is half of the native HRC pixel scale, and a resolution of $\sim 0.05^{\prime \prime}$ for point sources, one of the highest-resolution images obtained with HST.
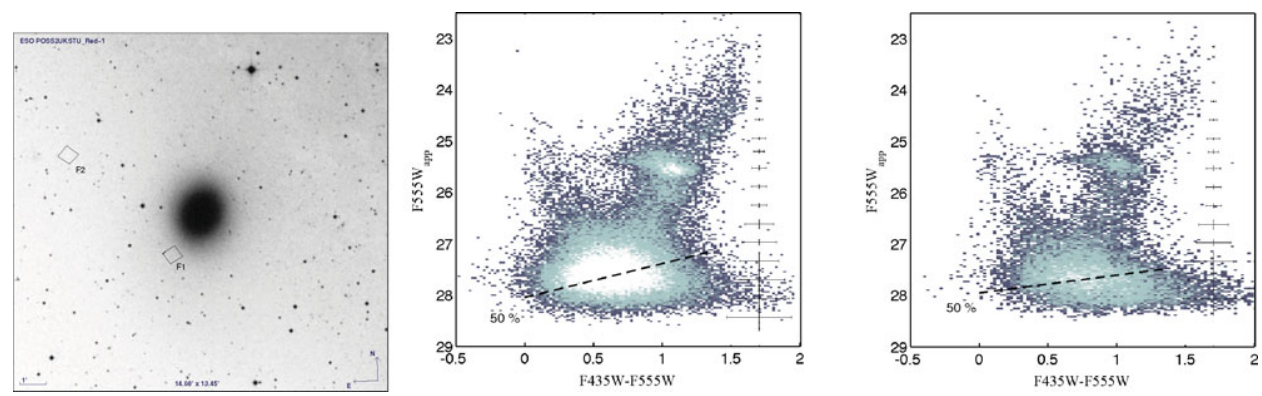

Figure 1. Left: Location of our two HST ACS/HRC pointings: M32 (F1) and M31 background (F2) fields. Each field covers a region of $26 \times 29 \operatorname{arcsec}^{2}$ on the sky. Middle: Hess representation with a logarithmic stretch of the CMD obtained from deconvolution of the images of the F1 field. The magnitudes are calibrated onto the HST VEGAmag system. The $50 \%$ completeness level is indicated by the dashed line and the photometric errors are shown in the right side of the figure. Right: Same as the middle panel for the F2 field.

Stellar photometry on the combined images has been performed in two different ways. In the first case, we use the standard DAOPHOT II/ALLSTAR packages (Stetson 1987). We build a PSF for each combined image and perform PSF fitting to obtain the stellar fluxes. In the second case we deconvolve the combined images and then perform aperture photometry by adding up light in $3 \times 3$ pixel boxes around the peaks on the deconvolved images. The results given by both methods are similar but the photometry performed on the deconvolved images is superior to that obtained using DAOPHOT II: the deconvolved CMDs show less scatter, thus producing notably sharper features at all luminosities. Furthermore, a visual inspection of the subtracted images reveals that the deconvolution does a considerably better job in resolving blended stars. We therefore use the CMDs from the deconvolved images for further analysis. Before the analysis of the CMDs, 
we performed exhaustive artificial star tests (ASTs) on F1 and F2 to understand the completeness of our data, which is mostly affected by crowding, as well as to quantify photometric errors. Fig. 1 (middle and right panels) shows the F1 and F2 CMDs obtained from the deconvolved images in a Hess representation. The dashed line indicates $50 \%$ completeness level at $F 555 W \sim 27.5$ for $\mathrm{F} 1$ and $F 555 W \sim 28$ for F2, and photometric errors obtained with ASTs are shown in the right side of each CMD. Finally, we correct the CMD in F1 for M31 stars by assuming that the population of M31 stars is statistically the same in both F1 and F2 fields and taking into account their different crowdings. A detailed description of these steps can be found in Monachesi et al. (2009) (hereafter M09).

Unfortunately, even though we have the highest resolution and deepest data for M32 yet obtained, the crowding is so severe that the observation of the oldest MSTO of M32, situated below the $50 \%$ completeness level of our data, still remains a challenge.

\section{Colour-Magnitude diagram analysis: Stellar populations of M32}

The proximity of M32, combined with the high resolution of HST ACS/HRC allows a remarkable improvement in our study of its stellar content. We can qualitatively study the stellar populations of M32 by comparing our CMD with theoretical isochrones. In the following, we assume a distance modulus of $\mu_{0}=24.50$ (M09) and a reddening of $E(B-$ $V)=0.08$ (Burstein \& Heiles 1982). We use theoretical isochrones from Padova library (Marigo et al. 2008; Girardi et al. 2002) as they are available in the HST ACS/HRC photometric system at different ages and metallicities.

Young populations (ages $<2$ Gyr): Fig. 2 (left panel) shows isochrones for young ages superimposed to the already decontaminated, de-reddened M32 CMD. The isochrones have metallicity $[\mathrm{Fe} / \mathrm{H}] \sim-0.37$ and ages of $0.3,0.5,0.7,0.9$, and $1.1 \mathrm{Gyr}$. This comparison suggests that M32 contains stars as young as $\sim 0.5-1.5 \mathrm{Gyr}$ and that any significant presence of populations younger than $0.3 \mathrm{Gyr}$ is ruled out in this field. Note that the "blue plume" (BP), not claimed before, remains in M32 even after decontamination for M31 stars. In addition we tested, using the results from ASTs, whether this BP could be actually artifact of crowding and we found that $\sim 80 \%$ are genuine blue stars (M09). The presence of a blue loop (BL) is another sign of young populations and has never been detected before in M32. Both BP and BL are indicated in Fig. 2 (left panel) as boxes.
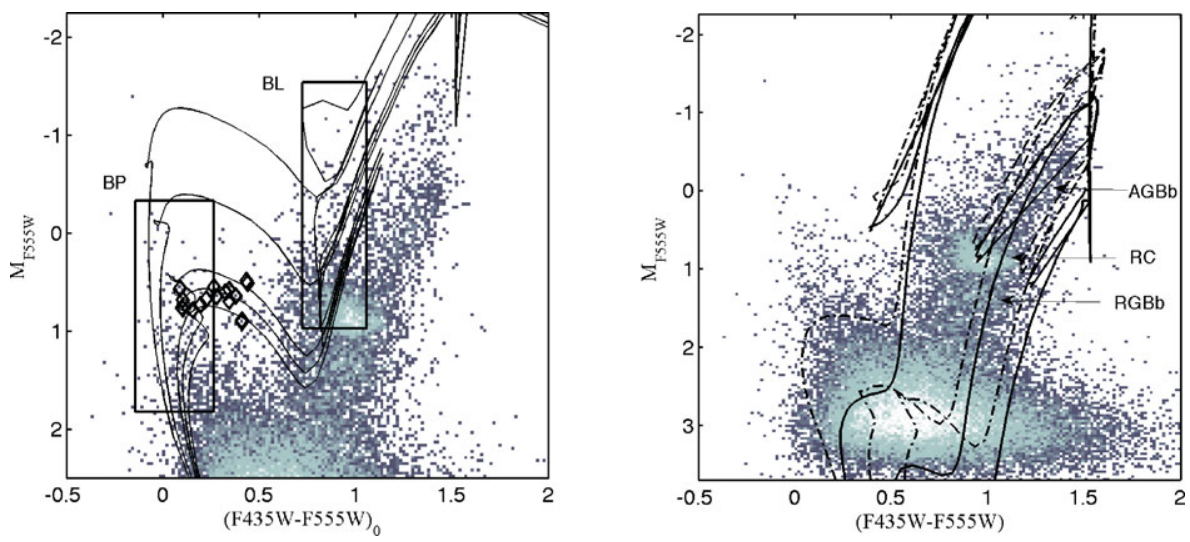

Figure 2. Absolute decontaminated M32 CMD in a Hess representation. Left: Isochrones of ages $0.3,0.5,0.7,0.9$ and $1.1 \mathrm{Gyr}$ for a $[\mathrm{Fe} / \mathrm{H}] \sim-0.37$ are overplotted. The $\mathrm{BP}$ and $\mathrm{BL}$ are shown in boxes. Diamonds represent the location of RR Lyrae found in F1. Right: Isochrones of 3 (dashed-dot) and 8 (solid) Gyr for $[\mathrm{Fe} / \mathrm{H}]=-2.27$ (bluest), -0.35 , and 0.2 (reddest) are superimposed. TheRC, RGBb and AGBb are indicated with arrows. See text for details. 
Intermediate age and old populations ( $2 \leqslant$ ages $<10$ Gyr): These are represented in our CMD by a strong red clump (RC), a red giant branch (RGB) and the red giant branch bump (RGBb). Extended and bright asymptotic giant branch (AGB) stars, identified here at $M_{F 555 W}<-1$, as well as the AGB bump (AGBb) are also produced by an intermediate-age population.

Fig. 2 (right panel) shows isochrones superimposed on the decontaminated CMD of M32, representing populations of 3 (dashed-dot isochrones) and 8 (solid isochrones) Gyr with metallicities of $[\mathrm{Fe} / \mathrm{H}]=-2.27$ (bluest), -0.35 , and 0.2 (reddest). Note that the RGB shows a rather wide colour distribution. Given the small photometric errors at these magnitude and colour levels, this cannot be explained by a single-age and -metallicity population but rather by an intrinsic large spread in the metallicity distribution, which has its peak at $[\mathrm{Fe} / \mathrm{H}] \sim-0.35$ (M09). If we solely look at the RGB, differences in ages cannot be distinguished. Fortunately, constraints on age and metallicity of these populations can be obtained from the mean colour and magnitude of the RC, as well as from the magnitude difference of each bump (RGBb and AGBb) with respect to the RC (Alves \& Sarajedini 1999, hereafter AS99). Theoretical isochrones predict, according to the locus of the $\mathrm{RC}$ in the $\mathrm{CMD}$, a mean age of $\sim 8 \mathrm{Gyr}$ and a metallicity of $[\mathrm{Fe} / \mathrm{H}] \sim$ -0.3. Moreover, according to AS99, the RC in our CMD located between a brighter AGBb and a fainter RGBb strongly indicates populations older than 2 Gyr and metallicities higher than about -0.7 dex. Given the AGBb-RC magnitude difference, the predictions by AS99 suggests that M32 metallicity is likely to be higher than $[\mathrm{Fe} / \mathrm{H}] \sim-0.5$ dex regardless of age. If we assume such a metal-rich population, the $\mathrm{RBGb}-\mathrm{RC}$ magnitude difference suggests that the mean age of M32 is likely to be $\sim 8.5 \mathrm{Gyr}$, which agrees with the value above from the $\mathrm{RC}$ alone.

Ancient populations (ages $\geqslant 10 \mathrm{Gyr}$ ): They are represented by a well-populated RGB as well as blue extended HB (EHB) stars. The fact that there is not a noticeable presence of blue horizontal branch (BHB) stars in our CMD suggests that a well-developed old, metal poor population is unlikely to be present in M32. Nonetheless, RR Lyrae variable stars found in F1 with our data (Fiorentino et al. 2009) expose the presence of BHB stars in M32. Their location is shown as diamonds in Fig. 2 (left panel). From the metallicity distribution function of M32 in our field (M09), we estimated that these ancient and metal-poor stars represent $11 \%$ of the total light ( $9 \%$ of the total mass) in our field.

\section{References}

Alves, D. R. \& Sarajedini, A. 1999, ApJ, 511, 225

Burstein, D. \& Heiles, C. 1982, AJ, 87, 1165

Coelho, P., Mendes de Oliveira, C., \& Fernandes, R. C. 2009, MNRAS, 396, 624

Davidge, T. J. \& Jensen, J. B. 2007, AJ, 133, 576

Fiorentino, G., Monachesi, A., Trager, S. C., Lauer, T. R., Saha, A., Mighell, K., Freedman, W. L., Dressler, A., Grillmair, C. J., \& Tolstoy, E. 2009, ApJ, submitted

Girardi, L., Bertelli, G., Bressan, A., Chiosi, C., Groenewegen, M. A. T., Marigo, P., Salasnich, B., \& Weiss, A. 2002, A\&A, 391, 195

Grillmair, C. J. et al. 1996, AJ, 112, 1975

Lauer, T. R. 1999, PASP, 111, 227

Marigo, P., Girardi, L., Bressan, A., Groenewegen, M. A. T., Silva, L., \& Granato, G. L. 2008, $A \mathscr{E} A, 482,883$

Monachesi, A., Trager, S. C., Lauer, T. R., Freedman, W. L., Dressler, A., Grillmair, C. J., \& Mighell, K. 2009, ApJ, submitted

O'Connell, R. W. 1980, ApJ, 236, 430

Rose, J. A. 1985, AJ, 90, 1927

Stetson, P. B. 1987, PASP, 99, 191

Trager, S. C., Faber, S. M., Worthey, G., \& González, J. J. 2000, AJ, 120, 165 